\title{
Design and Implementation of Distributed Cognitive Medium Access Control Protocol for Multi-hop Mobile Ad Hoc Network with Single Transceiver
}

\author{
Qingchao Zhü ${ }^{1,2, a,{ }^{*}}$, Jing Chen ${ }^{2,3}$, Mingfei $\mathrm{Liu}^{1}$, Lin Dong ${ }^{1}$, Ruichao $\mathrm{Hu}^{1}$ \\ ${ }^{1}$ School of Information Engineering, Engineering University of Chinese Armed Police Force, Xi'an, \\ Shanxi 710086- China \\ ${ }^{2}$ School of Information and Navigation, Air Force Engineering University, Xi'an, Shanxi 710077- \\ China \\ ${ }^{3}$ Department of Information Engineering, Air Force Xi'an Flight Academy, Xi'an, Shanxi 710300- \\ China \\ *atgzy0516zqc@126.com
}

Keywords: mobile ad hoc network (MANET), cognitive medium access control (MAC) protocol, power saving mode (PSM), channel sensing; channel vacate time (CVT), channel opening time (COT)

\begin{abstract}
Recently, cognitive medium access control (MAC) protocol has played a dominant role in mobile ad hoc network (MANET). However, existing algorithms ignore mobility, multihop nature and channel selection fairness. Thus extension of them becomes a critical issue. In this paper, we attempt to address the improvement of these shortages based on power saving mode (PSM) by redesinging channel sensing and data transmission for nodes deployed with a single transceiver. The contribution of this paper is threefold. First, we present the flowchart of proposed model with announcement traffic indication message (ATIM) and DATA window, resulting in separation of control channel and data channel in time field. To realize it, three types of channel information as state, maintenance time and selection priority are raised, based on which channel sensing, information sharing and channel sorting are realized. Second, we realize channel sensing and state sharing through redefinition and cooperation of control packets to record channel state, idle or busy. Third, we propose multi-hop cooperation, channel state updating/changing and reservation mechanisms to exchange packets, guaranteeing that channel would be used fairly and balanced. Simulation results show that, not only throughput and channel utilization are improved as well as acceptable value of channel vacate time (CVT) and channel opening time (COT), but also multi-hop, mobility and fairness nature are taken into account, which meets the need of MANET.
\end{abstract}

\section{Introduction}

Mobile ad hoc network (MANET) is an autonomous wireless networking system consisting of independent nodes without fixed or static infrastructure, which play a role as both terminal and router [1]. Aiming at limited bandwidth, cognitive medium access control (MAC) protocol has been focused for several years $[2,3]$. However, they are not suitable to MANET for lack of critical factors taken into consideration like multi-hop, mobility and channel fairness/balance.

As everyone knows, multi-hop transmission is different to single hop with constant channels, since amount of channels in the former formation decrease because of intermediate nodes forwarding. Besides, mobilty produces time-varing and random parameters of channels, meaning that, connections will appear/disappear along with entering or leaving the range of neighbor nodes, which causes retransmission or higher packet loss rate. Moreover, channel selection fairness, independent with misbehavior, means channel is chosen in decending order of index, not taking payload into account at all, resulting in unbalanced utilization of channels and low throughput as well. Therefore, how to improve them is an critical issue facing now.

Previous works have revelled that mobility is relative to receiving power, that multihop may be solved through channel cooperation, and that selection fairness could be optimized by redefinition of 
channel priority[4,5]. For the above-menthioned reasons, in this paper, focusing on taking multi-hop, mobility and fairness into consideration, we will design a novel cognitive MAC protocol to tackle the above issues in three steps. First, we propose a novel framework based on PSM mechanism[6] with ATIM and DATA window, which realizes separation of control channel and data channel in time field by raising three types of channel information as state, maintenance time and selection priority, based on which channel sensing, information sharing and channel sorting are acheived. Second, we realize channel sensing and information sharing by defining control packets to record channel state as idle or busy. Third, we propose multi-hop cooperation, channel state updating/changing and reservation mechanisms to exchange packets, guaranteeing that channel would be used fairly and balanced. Our results show that, both throughput and channel utilization are improved with acceptable CVT and COT[7]. Obviously, multi-hop, mobility and fairness are taken into account as well.

Specifically, the main contributions of this paper are the following:

1) framework design for MANET with a single transceiver with channel sensing and data transmission;

2) implementation of channel sensing based on channel state information, maintance time and priority including mobility;

3) channel reservation and cooperation of multi-hop together with fairness;

The remainder of this paper is outlined as follows. Section 2 summarized the related work. In Section 3, an novel model is proposed for multi-hop MANETs. Section 4 describes channel sensing and Section 5 presents data transmission. Simulation results are shown in Section 6. Section 7 concludes the paper.

\section{Related Work}

Cognitive radio(CR) $[2,8,9,10]$ could improve spectrum utilization efficiency as well as spectrum scarcity problem, making it one of the most intensively technology in MANET. It's widely used in MAC protocol like 802.11, 802.22, 802.19 and 802.15 standardized by IEEE group. 802.11 is widely applied in WSN, VANET, MANET and WRN and so on, so that distributed coordinate function (DCF) protocol based on CSMA/CA is focused[11], based on which many improved models have been proposed, such as CMAC, HC-MAC, MMAC-CR[12].These protocols address simlar issues, operating in a multi-channel context and facing multichannel hidden terminal problem. For example, Haythem et al [13] advocate a statistical approach used to allow communication opportunistically guaranteeing the performance of PU aiming at allowing CR users to share the licensed spectrum with PU without degrading the throughput of the latter. Ossma et al [14] present that popular simulation tools are not ideal for prodiding designer with guidelines to select and tune parameters when mission is in progress, so that new challenges of automated design and reconfiguration are faced. They analyze concepts of reconfiguration, describe design objectives and implementation options. Przemyslaw et al [15] give several observations which provide a concept of CR MAC design as follows. Throughput of PU will not decrease when control channel is implemented on a dedicated channel. Control packets exchange efficiency impact the achievable performance of PU. KANG et al [16] provides a comprehensive survey of cognitive radio technology on application of dynamic spectrum access based on CR technology and protocol stack, such as spectrum sensing, data transmision, security and CR MAC protocol, providing a basis to design novel model. Wha et al [4] point out that common control channel has influnce on throughput improvement, that opportunistic sensingis better than just before transmission sensing so that the former one will be used in this paper, and that propose a novel method to update channel state information as well as data transmission. Saptarshi et al [17] present that absence of infrastructure necessitaes the need for a contention based on MAC protocol where there can't be any prsumption on coordination without considering alailable channels varying with time. Focusing on it, they introduce the provision of reservation of free channels by secondaries for exteded periods to increase utilization without causing harmful interference to PU, whose correctness is demonstrated through performance metrics like blocking probability, secondary usage and degradation of PU. 
In addion, all the above-mentioned models have several common features. First, nodes deployed in MANET are configured with at least two transceivers to make seperation of control channel and data channel, but existing equipment has only one. Second, they perform sensing through certain nodes assigned at first, but there hasn't such nodes in MANET. Third, algorithms are limited to single hop, meaning that they are not suitable for multi-hop network at all. Last, none of them take mobility into consideration, which also differentizes with MANET.

Obviously, at first, power of nodes vary with time because of mobility, thus establishing relationship between them may make it more suitable than present models. And then how to extense channel cooperation and reservation to multihop network based on recent algorithms is a key issue. Forturnately, it may be realized based on channel state information discussed later, though making it a litter complex. Last, to reduce cooperation of multihop communication, channel selection improvement should be considered as well. For the above reasons, we will design a nove cognitive MAC protocol based on PSM mechanism.

\section{Proposed MAC Protocol}

To extend DCF protocol to multi-hop MANET of which nodes are equipped with a single transceiver, in this section, we first propose a novel framework including opportunistic sensing and data transmission based on PSM mechanism. Then to implement it and take multi-hop, mobility and fairness into account, state information, maintenance time $(\mathrm{Tm})$ and priority of channels are defined.

\subsection{Cognitive MAC Protocol for Multi-hop MANET with Single Transceiver}

First, several assumptions used to the novel MAC protocol are listed as followings.

- All nodes include $N$ available channels with the same bandwidth without overlapping, which guarantees that different channels will not interfere with others. Any node could obtain channel knowledge through cooperation with other hosts.

- Each node is equipped with a half-duplex transceiver so that it either transmits or senses, but couldn't realize both simultaneously.

- Transceiver could switch channels automatically, of which elapsed time is less than $224 \mu \mathrm{s}$.

- Nodes synchronization are realized based on the timing synchronization function (TSF) algorithm to make sure that frame could transmit and receive at same time.

Based on the above hypotheses, it's time to develop two vital components of cognitive MAC protocol called channel sensing and data transmission. However, they could not be able to realize through space separation by nodes for a single half-duplex transceiver. Instead, time field separation becomes an ideal method. Fortunately, PSM mechanism embedded in DCF protocol makes it possible. Therefore, distributed cognitive MAC protocol could be realized by combining cognitive capability with PSM. Thus, the final MAC protocol model for MANET is designed as Fig.1, which is similar to 802.22, with differences like absence of common control channel, single transceiver, considering natures of multi-hop, mobility and fairness (which are achieved by channel cooperation, packets transceiver power and channel update/selection discussed in the following sections respectively), etc. 


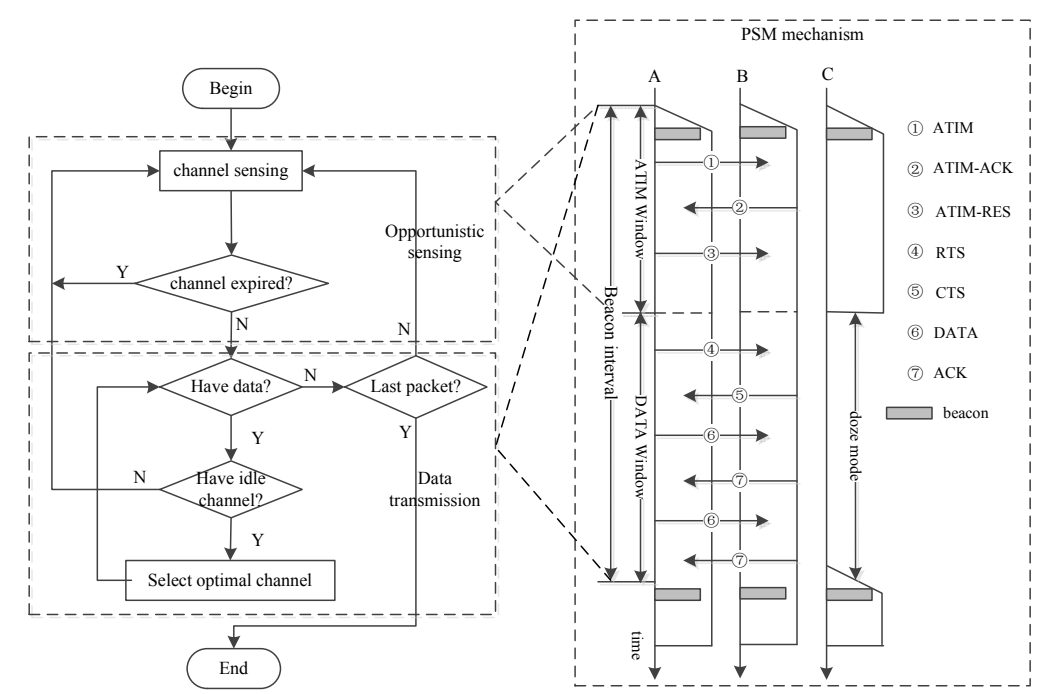

(a) Flowchart of algorithm precedure in novel model

Fig. 1 Cognitive MAC protocol model

(b) PSM mechanism

Fig.1 (a) and (b) illustrate the flowchart of algorithm procedure in novel model and corresponding PSM mechanism respectively. Obviously, in Fig.1 (a), the model is divided into two parts according to requirements of MAC protocol, which are known as opportunistic sensing and data transmission. The former is corresponding to ATIM window of PSM mechanism to achieve channel state information collection and sharing among nodes, all of which are relative to channel state information fields. Similarly, the latter utilizes entire beacon interval to implement channel reservation and data transmission, essential items of which consist of cooperation, of available channel and occupation duration distribution. The implementation of new model is shown in Fig.1 (b), where ATIM window and DATA window represent control channel and data channel respectively, both periods of which form a beacon interval. During ATIM window, channel sensing packets (RN_ON, RN_OFF and RN_REQ) and cooperation packets (ATIM, ATIM-ACK, ATIM-RES) are exchanged and all nodes maintain awake. However, in DATA window, data packets like RTS/CTS/DATA/ACK in virtual carrier sensing (VCS) mechanism are transmitted once data channel is confirmed.

For example, asuming that node A has shared available channel information with B to realize channel sensing. Later, if A has packets to transmit to B, A sends ATIM packet in ATIM window to fulfill channel cooperation at first. Then B select an idle channel whose number is embedded in ATIM-ACK for sender as well as notifying the rest available channels by broadcasting ACK-RES packets to its neighbor nodes. Specially, it's possible that several nodes may cooperate successfully for the same idle channel at a certain beacon interval. Therefore, VCS should be introduced to avoid collision. That is, A transmits RTS to contend for the channel. In return, B sends CTS once receiving RTS. Both RTS and CTS embed NAV to inform other neighbors to backoff. Similarly, DATA and ACK packets will be tranmitted in DATA window. Simultaneously, their neighbor node C keeps awake in ATIM window for channel sensing and dozen in DATA window to save power. It's obvious that cognitive MAC protocol realizes seperation of control channel and data channel in time field. However, it doen't take multi-hop, mobility and fairness into consideration, all of which would be focused in novel model by redefinition of reserved fields for sake of lessening overhead . To realize it and engage with channel, channel state information, maintenance time and priority will be defined at first.

\subsection{Channel State Information Fields}

Definition of channel state information is undipensable to ensure higher possiblity of successful transmission through interaction among nodes, relying on channel instantaneous state collection and share, like available channel number, activity of PU and cognitive (Cognitive Radio, CR) user etc. For this purpose, according to [4], several symbols representing mobility (energy) and multi-hop (channel) are defined as follows.

1) $C H_{-} S T_{k}(i)$ represents state of channel $i$ in node $k$, and $11 / 10$ indicate that PU/CR is active while 00/01 denote idle. 
2) $R N(i)$ represents responsibe node $i$, which observes PU or CR node in channel $i$ together with CH_ST $T_{k}(i)$.

3) $S(i)$ and $R_{k}(i)$ denote the moments that channel $i$ becomes idle and that any node $k$ could transmit packets.

4) $H_{k}(i)$ is a set of neighbor nodes of $k$, certain ones of which have discovered activity state of PU or CR in channel $i$.

5) $\varphi(x)$ is the vector of available channels in node $x$;

6) $T_{k}(i)$ is timestamp of channel $i$ sensed by node $k$.

7) $P_{k l}(i), P_{k n}(i), P_{m}(i), P_{k t}(i)$ denote the last received packet power between $\mathrm{k}$ and next hop, receiving packets' power at now, transmission power by node $\mathrm{k}$, and power threshold of receiver, through channel $i$ respectively. It's notable that $P_{k t}(i)$ is related to sensitivity of physical antenna. For simplifying, assuming that all nodes are configured with the same antenna, value of $P_{k t}(i)$ is equal at all nodes.

Based on channel state information defined above, for sake of considering mobility and multi-hop as well as fairness, channel priority is indispensable, which depends on descending order of maintenance time, both of which will be defined later. Intuitively, the proposed protocol performs better at cost of sacrificing storage computing resources in node, with little overhead introduced.

\subsection{Channel Maintenance Time}

Obviously, the poorer quality of channel chosen by any node to realize opportunity sensing or data transmission, the higher probability of link interruption together with packet loss will be. To achieve expected quality of communication, node performs retransmission constantly, resulting in heavy overhead introduced, which may block or even paralyze the whole network. Fortunately, channelsorting mechanism before selection is an efficient method to optimize it which should be suitable for MANET. Therefore, to characterize mobility, we define channel maintenance denoted by $T_{m}$ as a priority indicator, by which all channels are arranged in descending order, and its definition is as follows.

Definition $T_{m}$ equals meaning time from current power to threshold $P_{k t}(i)$. Since node velocity and channel parameter change frequently, calculus should be adopted for computation of $T_{m}$, while computation delay is introduced. As sensing period is much less than data transmission during which sudden change probability of velocity is so small that velocity is regarded as constant. Furthermore, receiving power is a positive correlation function with it, so the former varies linearly which simplifies the design of cognitive mac protocol. We assume the ratio of power change as a constant value $P$ in any sensing period. Let $T_{s}$ denote sensing interval. $T_{s}$ can be formulated as $\left(P_{k l}(i)-P_{k n}(i)\right) / T_{s}$ while nodes move away from each other, and opposite value is $\left(P_{k n}(i)-P_{k l}(i)\right) / T_{s}$, so

$$
P^{\prime}=\frac{\left|P_{k l}(i)-P_{k n}(i)\right|}{T_{s}}
$$

It could be divided into two parts. The one is that when nodes move close to each other, $T_{m}$ equals to sum value of $P_{k n}(i)$ to maximum power and $P_{k n}(i)$ to $P_{k t}(i) . T_{m}$ is fomulated as

$$
T_{m}=\frac{\left(P_{m}-P_{k n}(i)\right)+\left(P_{m}-P_{k t}\right)}{P^{\prime}}=\frac{2 P_{m}-P_{k n}(i)-P_{k t}}{P^{\prime}}
$$

The other is that when the nodes move away from each other, $T_{m}$ equals to the value of $P_{k t}(i)$ to $P_{k t}(i)$, that is

$$
T_{m}=\frac{P_{k n}(i)-P_{k t}}{P^{\prime}}
$$

By substituting (1) into (2) and (3), we can obtain $T_{m}$, and channels could be sorted in descending order of $T_{m}$, that is, the bigger $T_{m}$ is the lower interruption probability is and the higher probability it will be chosen. Therefore, it avoids blindness of channel selection.

However, certain problem occurs simultaneously. For instance, if power embeded in packets is transmitted in form of float type directly with 4 Bytes overhead introduced, which is not neligible to packets, how to deal with it becomes the key. We handle it according to quantization and coding mechanism, whose feasibility and validity have been demonstrated in telecommunications field [18]. 
When receiving power is lower than minimum transmission power $P_{\min }$, that is, $T_{m}$ is less than transmission time needed, all power values among $\left(0, P_{\min }\right)$ are meaningless. On contrast, the higher transmission power is, the longer $T_{m}$ is until the maximum value $P_{\max }$. Therefore, power value is divided into seven segments with application of non-proportional quantization technique, each of which will be encoded through Gray mechanism for security considerations as shown in Fig.2.

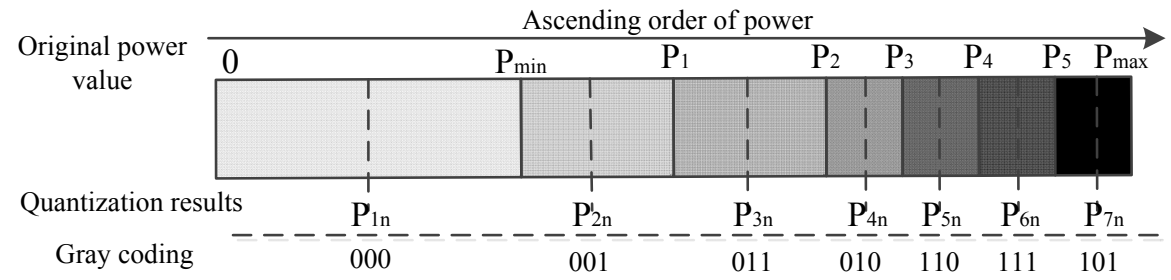

Fig. 2 Diagram of power value coding

We can observe that power value is expressed in 3 bit represented by $\mathrm{P}_{1} \mathrm{P}_{2} \mathrm{P}_{3}$, overhead is decreased by $91 \%$, which makes it possible to reuse and redefine reserved bits for receiver estimating $T_{m}$ and confirming the order of channel priority.

\subsection{Channel Priority}

Channel priority should include two factors, timestamp and $T_{m}$. The former represents counter value of storing time for channel information. The smaller it is which illustrates the longer it has been written, the higher expiration probability is, and the lower credibility and priority will be. The latter expresses extension how closer a node become to each other. The smaller $T_{m}$ is, the longer distance between nodes is which results in higher probability of link interruption as well as the smaller probability of successful transmission, and the lower priority is. Therefore, priority of the $i$ th channel in node $k$ is defined as

$$
c h_{p}=\left\{t-T_{l}\right\} / f\left(T_{m}\right)
$$

The smaller $c h_{p}$ is, the higher priority of channel is. In eq.(4), $f\left(T_{m}\right)$ is a monotone increment function of $T_{m}$, and we adopt $f\left(\left(T_{m}\right)\right)=2^{\left[T_{m}\right]},[*]$ is integer function. Specially, we emphasize two points about it, The one is enable parameter $e_{t}$ is introduced to avoid resource wastage caused by frequent sensing, that is sensing is executed only when $T_{l}$ is greater than $e_{t}$ expressed as $t-T_{l} \geq e_{t}$. The other is, $f\left(T_{m}\right)$ is greater than $t-T_{l}$, normalization method is employed to avoid errors caused by little numerator. Thus eq. (4) is redefined as

$$
c h_{p}=\lambda\left\{t-T_{l}\right\} / f\left(T_{m}\right), t-T_{l} \geq e_{t}
$$

where $\lambda$ is weighting coefficient. Obviously, eq. (5) considers both expiration and mobility on channel selection.

\section{Channel Sensing Cooperation}

Target of channel sensing cooperation is to update and share channel state information through control packets in ATIM window. It's achieved only when transceiver becomes idle according to channel information defined before. Then all channels will be sorted in ascending order of $c h_{p}$, which will be broadcast by responsible node in form of channel management packet. To keep pace with PSM in DCF, we still adopt ATIM, ATIM-ACK and ATIM-RES as packet formations, which will also be used in data transmission later. To distinguish them from different intervals, we set a flag in DCF header. Thus, they will be rewritten as RN_ON, RN_OFF and RN_REQ in channel sensing if $\mathrm{FLAG}=0$, otherwise be overwritten as RREQ, RRSP and $\overline{C H R E L}$ if $F L A G=1$ in data transmission.

\subsection{Channel Sensing initialization}

Nodes who are in charge of broadcasting channel information to others are called as responsible nodes(RN), with abilities of listening to PU, monitoring channel state, configuring sensing time, to avoid interference between nodes, which is to reduce sensing conflict and packet collision. Obviously, equation $R N(i)=N U L L$ characterizes that channel $i$ is occupied by PU or SU but not listened. Therefore, if node $k$ observes that packets are transmitted in channel $i$, it sets $R N(i)=\mathrm{k}$ and stores $i$ into channel 
list by descending order of $T_{m}$ or $c h_{p}$, which is expressed as $\mathrm{CH}_{-} S T_{k}=1, R N(i)=k, T_{k}(i)=$ Now . To avoid repeated setting of RN in other nodes, $k$ broadcasts RN_ON to notify other nodes updating information in form of $R N=k, S N=i, N A=j, C H=i, T_{k}(i)=N o w$, representing that $\mathrm{RN}$ is $k$, last hop is $i$, and next hop is $j$. To reduce confliction probability of sensing, $k$ sets quiet finish time (QFT) in each interval, which means that any node $n$ constructs RN_OFF with $N A=k, C H=i, T_{k}(i)=$ Now to $k$ once confirming state of channel $i$, after which $k$ cancels $\mathrm{RN}$ as $R N(i)=N U L L$ immediately and broadcasts it to neighbor nodes, which would executes opportunity sensing after a period of QFT. Furthermore, $k$ deletes $i$ from channel list if $T_{m}$ is less than the time required for packets transmission to reduce the probability of packet loss rate. Moreover, $k$ broadcasts sensing request packet RN_REQ with $C_{-} S_{T_{k}}=0, N A=N U L L, C H=i, R N(i)=N U L L$ while timestamp is larger than $e_{t}$ which illustrates that channel state information is unreliable because of expiration. However, it should set itself to be RN for lack of $k$ and fulfill the mentioned procedures proposed above.

\subsection{Interation of Control Packets}

Nodes will execute relate operations after receiving different channel management packets through multi-hop forwarding. Any node $j$ checks local channel list to decide whether there is $k$ as $\mathrm{RN}$ or not after receiving RN_ON. If not, $j$ set $\mathrm{k}$ as RN and insert $i$ into channel list based on the descending order of $T_{m}$, that is $R N(i)=k, C H=i$. Otherwise, it compares $T_{k}(i)$ with current time to judge its expiration, leading in two results. One is that node set $k$ as $\mathrm{RN}$ if the latter is not timeout with value greater than $T_{k}(i)$. The other is that node remains unchanged, and updates $T_{k}(i)$.

When node receives RN_OFF, $j$ unsets $k$ as $\mathrm{RN}$ with $R N(i)=N U L L, C H=i$. And then $i$ will be chosen as a candidate channel to sense after QFT.

When node receives RN_REQ, $k$ is set to be RN only if $T_{m}$ of $\mathrm{i}$ is larger than time to transmit. Otherwise, it forwards RN_REQ later.

\subsection{Channel Information Update}

Node $\mathrm{k}$ sets $\mathrm{CH}_{-} S T_{k}$ as $1 \mathrm{x}$ once detecting PU or CR after fulfilling opportunity sensing on channel .i. Otherwise, it is set to $0 \mathrm{x}$. For one aspect, when $C H_{-} S T_{k}$ equals to $1 \mathrm{x}$, value of $R N(i)$ field should be discussed to determine whether it is set as RN. If it is, ${ }^{T_{k}(i)}$ is rewritten while nothing is done if not. When $\mathrm{CH}_{-} S T_{k}$ is $0 \mathrm{x}$, packet transmission period is computed as well as channel listening in ATIM window but doze mode remains in data window till implementation of communication.

\section{Data Transmission}

Obviously, essence of cognitive MAC protocol is data transmission consist of channel reservation and packets exchange. The former acheives channel cooperation in ATIM window, corresponding to control channel, while the latter is corresponding to data channel to fulfill data transmission based on VCS in data window of reserved channel.

\subsection{Channel Reservation}

\subsection{Channel reservation and control packet design}

Channel reservation contains three steps in ATIM window like request, acknowledge and release, which are denoted by reservation request (RREQ), reservation response (RRSP) and channel release (CHREL)packets, respectively, illustrated in Fig.3.

RREQ, RRSP and CHREL cooperate among nodes in ATIM window. First, SA transmits RREQ including $n$ candidate channels with state information tag, written in $\mathrm{CH}_{-}$MAP field in descending order of $T_{m}$. For example, if tag is set to 0 , corresponding channel is busy; otherwise, it's idle. Then NA updates local channel state information once receiving RREQ, that is, if $i$ th tag of CH_MAP is 1 , node $k$ writes SA into $H_{k}(i)$, so $i$ is marked busy in $k$; otherwise SA is removed. Equation $H_{k}(i)=\{\}$ indicates that none of nodes select $i$ as data channel. Third, NA looks up local channel list in descending order of $T_{m}$ to determine whether same channels exist. If result is true, NA select it as data channel denoted by SEL_CH, send to SA in form of RRSP; otherwise, NA performs opportunity 
sensing when transceiver is idle till link is established. Last, when transmission is complete, SA or NA constructs CHREL to release REL_CH.

It is noteworthy that for MANET, the channel cooperation should take multihop and mobility into account as topology illustrated in Fig.4.-(a) and detailed algorithm in Fig.4.-(b). Results of channel negotiation for $\mathrm{A}$ and $\mathrm{E}, \mathrm{E}$ and $\mathrm{F}$ are 3 and 2 respectively.
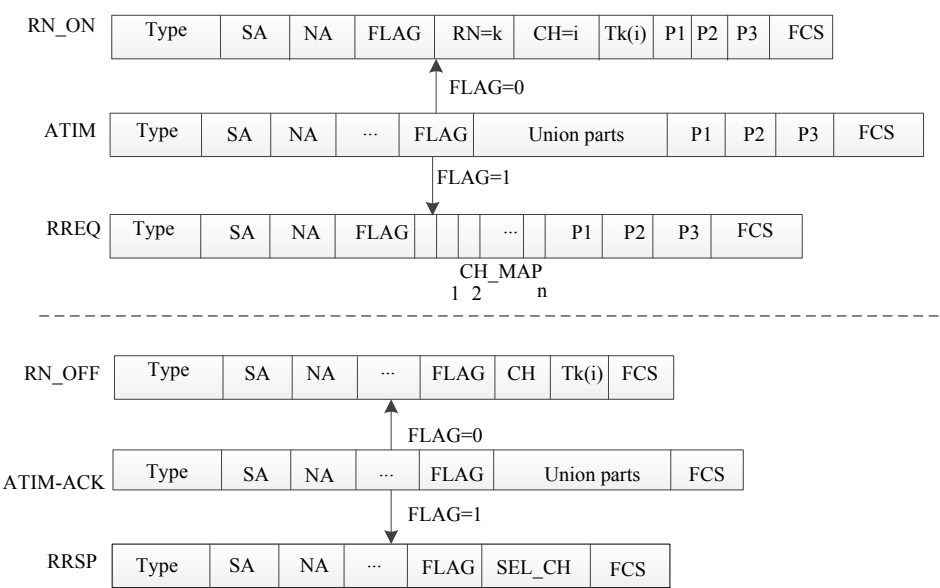

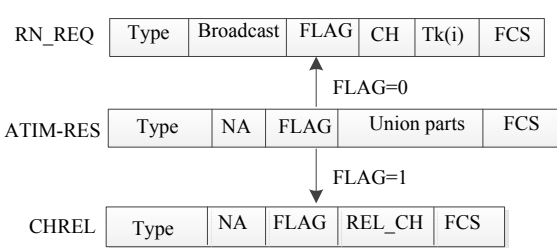

\begin{tabular}{c|c|c|c|c|c|c|}
\hline RTS & Type & RA & SA & DUR & $\cdots \cdots$ & FCS \\
\hline
\end{tabular}

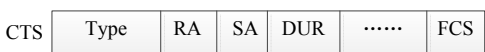

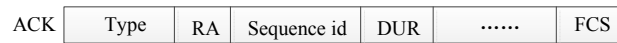

SA: Source address NA: Next address DUR: Duration of packets SEL_CH: Selected channel REL_CH: Release channel

Fig. 3 Formations of different packets

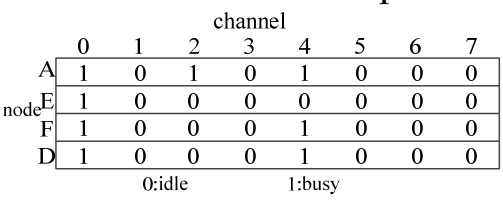

(2) Chanel selection is in descending order of Tm Before sorting After sorting \begin{tabular}{|ccccc|}
\hline 1 & 3 & 5 & 6 & 7 \\
\hline 3 & 5 & 1 & 7 & 6 \\
\hline
\end{tabular}

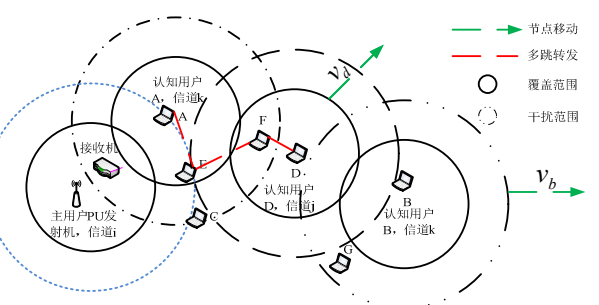

(a) Multichip forwarding network

(4) Fetch common channel for $\mathrm{E}$ and $\mathrm{F}$

$\boldsymbol{\varphi}_{E F}=\boldsymbol{\varphi}(E) \mid \boldsymbol{\varphi}(F)$ $=10011000$ consideration.
(1) Fetch idle channel between A and E

$\boldsymbol{\varphi}_{A E}=\boldsymbol{\varphi}(A) \mid \boldsymbol{\varphi}(E)$ $=10101000$

(3) Compute residue available channel

$\boldsymbol{\varphi}(E)=\boldsymbol{\varphi}_{A E}-3$

$=10010000$

(5) Channel is sorted in descending order via Tm

Before sorting \begin{tabular}{llllll|}
\hline 1 & 2 & 5 & 6 & 7 \\
\cline { 2 - 6 }
\end{tabular} \begin{tabular}{llllll} 
& After sorting & 7 & 5 & 1 & 6 \\
\hline
\end{tabular} Channel 2 is selected between $\mathrm{E}$ and $\mathrm{F}$

(6) Channel 3 and 2 are selected as data channel to transmit data in data window. Then selection and remainders are broadcast to neighbor nodes. Therefore, it takes mobility and multi-hop into

(b) channel cooperation algorithm

Fig. 4 proposed channel cooperation algorithm based on any multihop network

\subsection{Duration computation}

The essence of channel reservation is to compute reservation duration(denoted by DUR). Since it's possible that multiple couples may negotiate with a channel successfully at same time, we adopt VCS mechanism [6] to avoid collision, shown in Fig.5, based on which reservation duration is calculated.In fig.4, A and B represents source and destination as well as $\mathrm{C}$ to be monitor. $\mathrm{t}_{\mathrm{A}}$ and $t_{2}$ denote moments of channel reservation and completion of transmission.

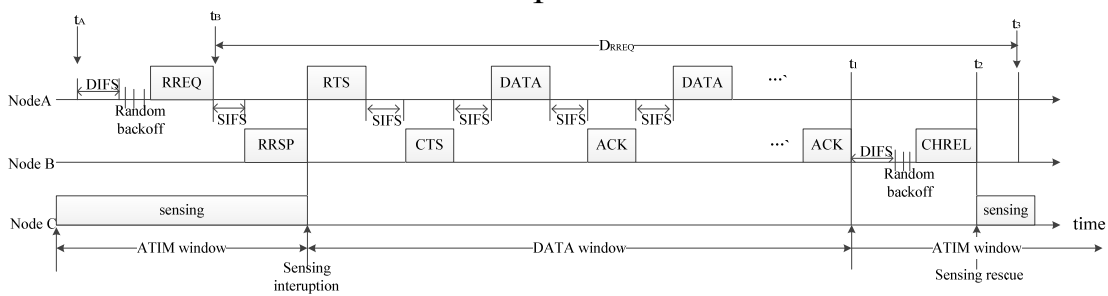

Fig. 5 VCS mechanism

DUR is computed as follows. Two conditions should be satisfied before channel reservation, that is, $\mathrm{A}$ has available channels and transceiver of $\mathrm{B}$ is idle. The former is true if $C H_{-} S T_{A}(i)=1 x, R_{A}(i)<t_{A}$ while the latter is determined by listening to RREQ/RRSP/CHREL packets of SA or NA in A. Assuming 
that transceiver of B is idle at $\Gamma_{B}$, that $T_{x}$ represents the moment of transmitting control packet $x$, and that length of SIFS and DIFS are denoted by $T_{\text {SIFS }}$ and $T_{D I F S}$, transceiver of $\mathrm{B}$ is idle when $\Gamma_{B} \leq t_{A}+T_{D I F S}+T_{\text {SIFS }}+T_{\text {RREQ }}+T_{R R S P}$ from Fig.5.

If both conditions are standing, A constructs RREQ with ordered channels of $\mathrm{C}_{-} \mathrm{CH}, \mathrm{C}_{-} \mathrm{CH}_{1}, \cdots, \mathrm{C}_{-} \mathrm{CH}_{n_{c}}$. If amount of available channels is less than $n_{c}$, the insufficient proportion is initialized to null. Thus, DUR represents an estimation of the period from sending RREQ to finishing data transmission. B compares $\mathrm{C}_{-} \mathrm{CH}$ field with local channel list in descending order of $T_{m}$ and delete those numbers whose $T_{m}$ is less than DUR after receiving RREQ. If they have common channels, B selects one as data channel to transmit packets based on CSMA/CA mechanism. Obviously, computation of DUR becomes an essential issue.

We analyze the DUR value in worst case, which has the maximum time from finish moment of channel sensing to finish moment of data transmission. Let $w$ denote channel number in the worst case, $\Gamma$ represents detection delay, the follow inequality is held.

$$
t_{A}+T_{D I F S}+T_{R R E Q}+D U R-l_{A}(w) \leq \Delta \times \Gamma
$$

Where $l_{A}(w)$ denotes the completion moment of sensing by A on $w . \Delta$ is the random compensation delay caused by false detection of PU, whose values is $0<\Delta<1$.

Let $R_{d f}$ denote default rate of packet transmission. All packets are sent with same length out. Let $V_{j}$ represent duration of single packet transmission. From figure 4, we obtain

$D U R=T_{R R S P}+T_{R S}+T_{C I S}+2 \times T_{\text {IIIS }}+k \times\left(V_{\text {CIS }}+T_{A C K}+2 \times T_{\text {SIF }}\right)$

Let $T_{\text {cnt }}=T_{R R E Q}+T_{R R S P}+T_{R T S}+T_{C T S}$. Plugging (7) into (6), $k$ is formulated as

$$
k \leq \frac{\Delta \times \Gamma+l_{A}(w)-t_{A}-T_{c n t l}-T_{D I F S}-2 \times T_{S I F S}}{V_{d f}+T_{A C K}+2 \times T_{S I F S}}=k_{\max }
$$

If A has $m$ packets to $\mathrm{B}, k=\min \left\{k_{\max }, m\right\}$. Plugging it into (8), solution equals the maximum of DUR. Similarly, B checks variables of channels in C_CH field after receiving RREQ at $t_{\mathrm{B}}$,:

$$
\left\{\begin{array}{l}
C H_{-} S T_{B}(i)=0 \\
R_{B}(i)<t_{B} \\
t_{B}+D U R-l_{B}(i) \leq \Delta \times \Gamma \\
D U R \leq T_{m}
\end{array}\right.
$$

where $l_{B}(i)$ denotes finish moment of sensing by B. If eq. (9) is established, that is, needed channel $i$ exists, which will be written in SEL_CH of RRSP from B to A. To simplify computation, DUR of RRSP is equal to that of RREQ. Simultaneously, sensing results are written to CH_MAP field as well. If there isn't any channel satisfying (9), it means that no link could be established between A and B, just relying on forwarding.

\subsection{Data Exchange}

Packets among different nodes are transmitted based on CSMA/CA to mitigate collision because it's possible that multiple nodes may compete identical channel successfully at same time. However, from Figure 4(a), we observe that nodes with mobility of MANET result in leaving the coverage range of each other, which raises channel changing. Moreover, other idle channels are used for packet forwarding. Therefore, update of channel list is imperative, which means that two additional abilities should be accomplished. The one is update of channel list, and the other is channel switching. The former is fulfilled through insert sensing channel into local list by descending order of $T_{m}$, such as channel 5 in the Fig.6. For the latter, it is realized by sequential number, that is, the higher priority of channel is, the more frequency of selection will be. For example, in Fig 6, if channel 4 doesn't meet the needed, node checks channel 1 , then channel 3, and so on, until a successful channel 5 . The method could ensure optimal channel selected. However, the higher priority of channel is, the more frequent it is used and the lower probability of idle state it will be, which leads to higher collision rate in certain channel and lower usage of other channels, with waste of channel resources, unfairness, and lookup delay. To improve it, a simple modification is done. that is, we just choose next channel based on $T_{m}$ from the current one. For example, in Fig. 6, if channel 4 couldn't maintain communication at all, node switches to channel 5 directly. 


\subsection{Information update}

Neighbor nodes update their channel information by listening to RREQ/RRSP/CHREL, classified to three parts.

1) Monitor node $\mathrm{C}$ inserts SA into $H_{\mathrm{c}}(i)$ after receiving RREQ from its one-hop neighbor if $i$ th bit of CH_MAP is 1 ; otherwise, it deletes SA.

2) $\mathrm{C}^{-}$sets $S(i)=\max \left\{S(i), t_{C}+d\right\}$ after hearing RRSP at time $t_{\mathrm{c}}$ with $\mathrm{SA}=\mathrm{A}, \mathrm{NA}=\mathrm{B}, \mathrm{DUR}=d$, $\mathrm{SEL} \_\mathrm{CH}=i$. If $\mathrm{C}$ is one hop neighbor of $\mathrm{A}$ or $\mathrm{B}$, it configures $R_{C}(i)=\max \left\{R(i), t_{C}+d\right\}$. Moreover, if $i$ th bit of CH_MAP equals to $1, \mathrm{C}$ inserts SA to $H_{\mathrm{c}}(i)$ while removes if not.

3) C sets $S(i)=t_{2}$ once receiving CHREL with $\mathrm{SA}=1, \mathrm{NA}=\mathrm{B}, \mathrm{REL} \_\mathrm{CH}=i$. Moreover, $\mathrm{C}$ sets $R(i)=t_{2}$ if it is neighbor node of $\mathrm{A}$ or $\mathrm{B}$.

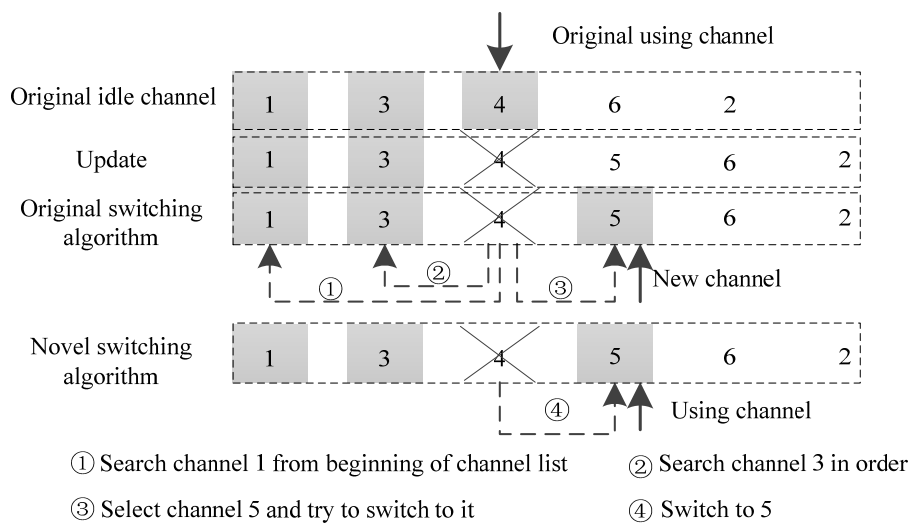

Fig. 6 channel information update and switch mechanism

\section{Numerical Results}

In this subsection, we analyze several metrics of cognitive MAC protocol, devided into two aspects, one of which incudes channel vacate time (CVT) and channel opening time(COT) representing interaction between PU and CR, the other of which incudes throughput and utilization ratio(CH_U) characterizing channel nature.

\subsection{Network settings}

Here, we adopt similar simulation model as those of previous work[19, 20] with unique difference of walk nodes, all of whose velocity are uniform assigned to $2 \mathrm{~m} / \mathrm{s}$ in the range of $5 \mathrm{~km} \times 5 \mathrm{~km}$. The mobility model of Random Walk is used. DSR (Dynamic Source Routing) protocol and CBR (Constant Bit Rate) traffic of 1450Bytes, whose transmission rates are 11Mbps, are set to network layer and application layer, respectively. Max number of connection to any node is set to 10 . Value of contention window is initialized to 32 . Each node equips with a transceiver, which could cover 16 channels. Other parameters of DCF standardized by IEEE 802.11 group are as shown in table 1 . We run our simulations for $600 \mathrm{~s}$, so that node distribution can reach a stable state. Each simulation and theoretical results are performed three instances and average values will be taken.

Table 1. Standard settings of DCF by IEEE 802.11 group

\begin{tabular}{|c|c|c|c|}
\hline parameter & value & parameter & value \\
\hline ACK expired time & $300 \mu s$ & Propagation delay & $1 \mu s$ \\
\hline CTS bits & $112 \mathrm{bit}+\mathrm{PHY}$ & slot length & $50 \mu \mathrm{s}$ \\
\hline CTS expired time & $300 \mu \mathrm{s}$ & DIFS & $128 \mu \mathrm{s}$ \\
\hline RTS bits & $160 \mathrm{bit}+\mathrm{PHY}$ & SIFS & $28 \mu \mathrm{s}$ \\
\hline Header bits & MAC:272bit PHY:128bit & ACK & $112 \mathrm{bit}+\mathrm{PHY}$ \\
\hline
\end{tabular}




\subsection{Results analysis}

Based on above settings, simulation results are shown in Fi.7 Fig.10.

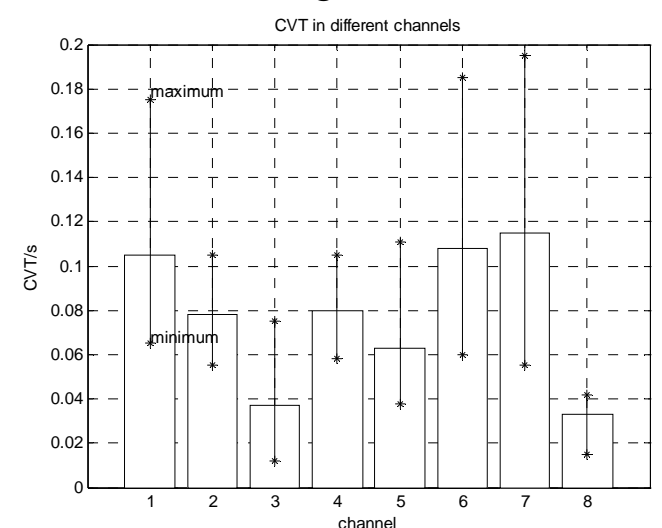

Fig. 7 Average CVT

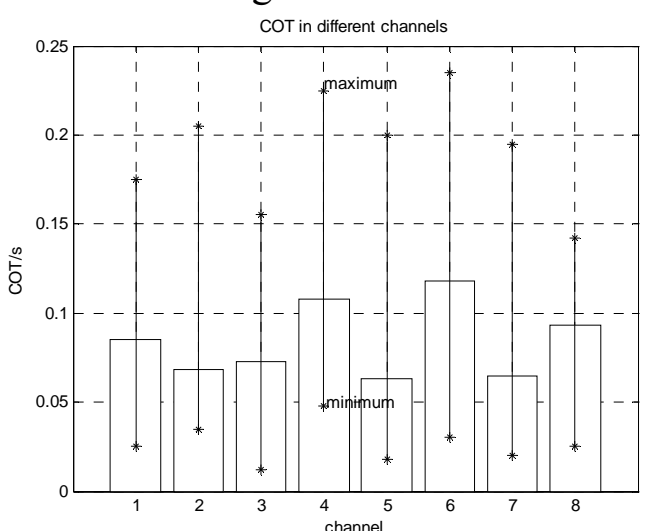

Fig. 8 Average COT

Fig. 7 and Fig.8 illustrate average CVT and COT, respectively. From Fig.7, in transverse comparison, we observe that CVT fluctuates according to the number of different channel. For example, CVT of channel 1 is $105 \mathrm{~ms}$, which is $70 \mathrm{~ms}$ higher than that of channel 8 , indicating influence to PU in channel 1 is more serious than channel 8, reasons of which comprise different periods (ATIM or DATA window) PU appears, varying type and size of packets(DATA is larger than ATIM control packets), etc. Moreover, in vertical comparison, there is a large deviation between maximum and minimum value of CVT, reasons of which are consist of usage frequency of channel as well as listening time of CR, etc. Similarly, COT has the same conclusion with a larger fluctuation, which means that PU is the object to guarantee for its authorization, so priority of the latter is higher even ignoring $\mathrm{CR}$.

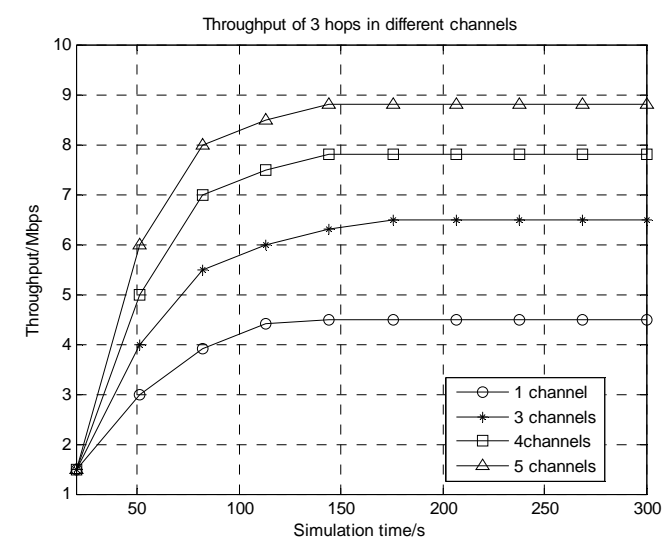

Fig. 9 Throughput of different channels in 3hops

Fig. 9 illustrates throughput growth with number of channels. Obviously, as number of nodes in MANET increase, throughput increase as well but not linearly, with raising range smaller and smaller. For each kinked line, throughput increases highly first, then stays a stable value because of network saturation. Reasons are that, more and more couples of nodes participate in transmission at first, and then maintained a saturated state, that is, total couples of transmit-receive nodes remain almost unchanged at all. 


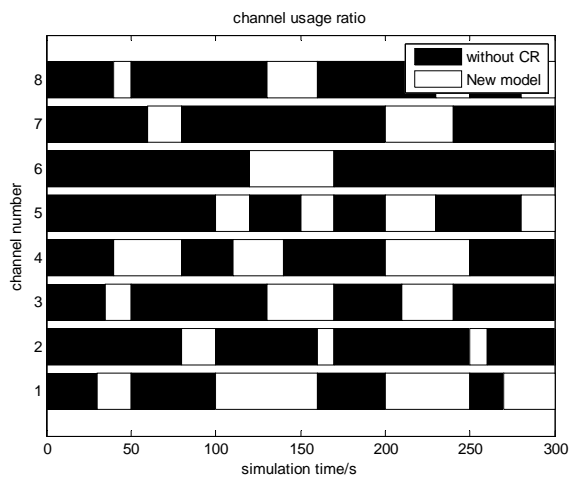

(a) Feathers not considered

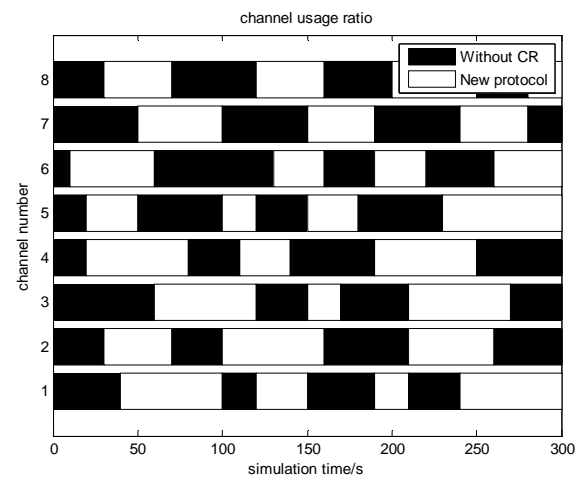

(b) Feathers considered

Fig. 10 Channel usage ratio

Fig.10 illustrates channel usage rate before and after introducing cognitive ability. Channel usage rate is higher in proposed MAC protocol (average usage time is about 150ms) than that without (only channel $1,3,4$ with time of $160 \mathrm{~ms}, 70 \mathrm{~ms}, 130 \mathrm{~ms}$ ) because many features are not considered at all, which proves efficiency and feasibility of the new protocol.

\section{Conclusion}

A novel cognitive MAC protocol is proposed based on PSM mechanism with consideration of mobility, multihop and channel selection fairness, including channel sensing and data transmission, responding to ATIM and DATA window, which seperates control channel and data channel in time field. The former is realized on basis of definition of channel state information as well as priority, while the latter is achieved, denpending on channel reservation and CSMA/CA, which pays attention to multihop and mobility. Results show that, COT and CVT are acceptable. Throughput and channel usage rate are both improved. However, there are still many items unconsidered, for instance, selfish misbehavior, physical sensing, multichannel hidden terminals, etc, which will be disscussed in future.

\section{Acknowledgements}

This work was jointly supported by the National Natural Science Foundation of China (No. 51075395, 61401499), the Industrial Science and Technology Project of Shaanxi Province (No. 2016GY-087).

\section{References}

[1]. B Kyoungsoo, Y Sooyong, L Jongtae and Y Jaesoo, "Grid based Enhanced Routing Scheme in MANET”, KSII Transactions on Internet and Information Sytems, vol.10, no.5, pp.2218-2244, 2016.

[2]. G Liljana, D Daniel, R Valentin, et al, "Medium Access Control Protocols in Cognitive Radio Networks: Overview and General Classification", IEEE Communication Surveys and Tutorials, vol. 16, no. 4, pp: 2092-2124.

[3]. D D Antonio, C S Emilio and D B Maria, "A Survey on MAC Strategies for Cognitive Radio Networks", IEEE Communication Surveys and Tutorials, vol. 14, no.1, pp:21-44, 2012.

[4]. S J Wha, A H Jeong and G J Dong, "A Novel MAC Scheme for Multichannel Cognitive Radio Ad Hoc Networks", IEEE Transactions on Mobile Computing, vol. 11, no.6, 2012.

[5]. P J Gyanendra, Y N Seung, A Srijana and W K Sung, "Analysis of Channel Access Delay in CRMAC Protocol for Ad Hoc Cognitive Radio Wireless Sensor Networks without a Common Control Channel," KSII Transactions on Internet and Information Systems, vol. 8, no. 3, pp. 911 923, 2014. 
[6]. IEEE Standard for Wireless LAN Medium Access Control (MAC) and Physical Layer (PHY) Specifications, Nov., 1997, P802.11.

[7]. T Michael, D Antoine, V D Liesbet and C Francky, "A Distributed Multichannel MAC Protocol for Cognitive Radio Networks with Primary User Recognition", In Proceedings of 14th Conference on Wireless Communication, 395-362, 2015.

[8]. Standard for Recommended Practice for Installation and Deployment of IEEE 802.22 Systems, IEEE 802.22.2-2012.

[9]. A N Mody, Z Lei, G Ko, et al, "Introduction to IEEE 802.11-2011 and Its Amendment PAR for 802.22: Broadband Extension and Monitoring”, IEEE 802 Plenary Session, 2011.

[10]. Draft Standard for TV White Space Coexistence Methods, IEEE P802.19.1 Draft, 2013.

[11]. D Eastlake 3rd and J Abley, "IANA considerations and IETF protocol and documentation usage for IEEE 802 parameters: RFC7042", available online at http:// www. rfceditor.org/rfc/rfc7042.txt.

[12]. C Cordero and K Challapali, "C-MAC: A Cognitive MAC Protocol for Multichannel Wireless Networks", In Proceedings of IEEE 11th International Conference on Trust, Security and Privacy in Computing and Communications, pp. 25-27, 2012.

[13]. A Haythem, M Marwan and Y Ossanma, "MAC Protocol for Opportunistic Cognitive Radio Networks with Soft Guarantees", IEEE Transactions on Mobile Computing, vol.8, no.10, 2009.

[14]. Y Ossanma, K Latha and Y Kenneth, "Cognitive MANET Design for Mission Critical Networks", Military Communications, pp: 64-71, 2009.

[15]. P Przemyslaw, P Sofie, W S Hoi and H Ramin, "Performance Analysis of Multichannel Medium Access Control Algorithms for Opportunistic Spectrum Access”, vol. 58, no.6, 2009.

[16]. G Kang, K Hyoil and K Ashwini, "Cognitive Radios for Dynamic Spectrum Access: From Concept to Reality”, IEEE Wireless Communications, 64-74, 2010.

[17]. D Saptarshi, D Swades and C Mainak, "Contention Based Multichannel MAC Protocol for Distributed Cognitive Radio Networks", IEEE Transactions on Mobile Computing, vol. 13, no. $12,2014$.

[18]. C X Fan, "Principles of Communications, Seventh Edition", 2013.

[19]. S T Geetam, S Tripti, B Debnath and T H Kim, "Performance Comparision of AODV, DSR and DSDV under Various Network Conditions: A Survey", in Proc. of International Conference on Ubiquitous Computing and Multimedia Applications, pp. 3-7, 2011.

[20]. M A S Ali and A A Bander, “Throughput comparison of AOMDV and OLSR Ad Hoc Routing Protocols Using VBR and CBR Traffic Models", in Proc. of International Conference on Advanced Computer Science Applications and Technologies, pp.466-469, 2013. 